\title{
Minimum radiative heat transfer between two metallic half-spaces due to propagating waves
}

\author{
A. Narayanaswamy ${ }^{\mathrm{a}, *}$, J. Mayo $^{\mathrm{b}}$ \\ ${ }^{a}$ Department of Mechanical Engineering, Columbia University, New York, NY 10027 \\ ${ }^{b}$ Skycatch Inc., San Francisco, CA 94107
}

\begin{abstract}
The gap dependence of radiative energy transfer due to propagating waves between two identical metallic half-spaces separated by vacuum is investigated. The dielectric function of the metallic half-spaces is described by the Drude model. Analytical expressions for the minimum radiative heat transfer coefficient, $h_{\min }$, and the gap, $d_{m i n}$, at which the minimum value of radiative transfer is attained are determined in terms of the parameters of the dielectric function and the absolute temperature $T$. We show that $h_{\min } \propto T^{2}$ in the high temperature limit and $h_{\min } \propto T^{7 / 2}$ in the low temperature limit.
\end{abstract}

Keywords: Radiative transfer, Interference effects

PACS: 44.40.+a, 41.20.-q, 41.20.Jb, 42.25.-p

\section{Nomenclature}

$\Delta F F \quad$ Relative change in $h_{t o t}$ relative to the far-field heat transfer coefficient $h_{f f}$

$\mathcal{T}_{j} \quad$ Polarization dependent transmissivity

$c \quad$ Speed of light in vacuum

$d \quad$ Thickness of vacuum gap between two half-spaces

$d_{\text {min }} \quad$ Vacuum gap at which $h_{p w}$ attains a minimum

$h_{f f} \quad$ Far-field radiative heat transfer coefficient

$h_{p w} \quad$ Radiative heat transfer coefficient due to propagating waves

$h_{p w}^{(l g)} \quad$ Contribution to radiative heat transfer coefficient from modes active at larger gaps

$h_{p w}^{(s g)}$ Contribution to radiative heat transfer coefficient from modes active at smaller gaps

$h_{\text {tot }}(d)$ Total (propagating and evanescent) radiative heat transfer coefficient at vacuum gap $d$

$k_{0} \quad \omega / c$, wavenumber in free space

$k_{B} \quad$ Boltzmann's constant

$k_{z 0} \quad z$-direction wavenumber in free space

$k_{z 1} \quad z$-direction wavevector in half-space

$n_{1} \quad$ Real part of $\sqrt{\varepsilon_{1}}$

$R_{j} \quad$ Polarization dependent Fresnel reflection coefficient

\footnotetext{
*Corresponding author

Email address: arvind.narayanaswamy@columbia.edu (A. Narayanaswamy)
}

$R_{j}^{a} \quad$ Approximation to $R_{j}$

$T \quad$ Temperature of half-spaces

$x \quad k_{z 0} / k_{0}$, non-dimensional wavenumber

$x_{n} \quad$ Non-dimensional wavenumber for $n^{\text {th }}$ spike in $\mathcal{T}_{j}(x, y)$

$z \quad$ Non-dimensional frequency, $\omega / \gamma$

$\epsilon_{\gamma} \quad$ Non-dimensional damping frequency, $\hbar \gamma /\left(k_{B} T\right)$

$\epsilon_{\omega_{p}} \quad$ Non-dimensional plasma frequency, $\hbar \omega_{p} /\left(k_{B} T\right)$

$\gamma \quad$ Damping frequency in Drude model of $\varepsilon(\omega)$

$\hbar \quad$ Planck's constant divided by $2 \pi$

$\kappa_{1} \quad$ Imaginary part of $\sqrt{\varepsilon_{1}}$

$\lambda$ Wavelength of electromagnetic wave in free space, $2 \pi / k_{0}$

$\omega \quad$ Angular frequency of electromagnetic wave

$\omega_{p} \quad$ Plasma frequency in Drude model of $\varepsilon(\omega)$

$\sigma \quad$ Stefan-Boltzmann constant

$\varepsilon \quad$ Dielectric function

$\varepsilon_{1} \quad$ Dielectric function of half-space

$\xi \quad$ Inverse of non-dimensional gap, $\left[\frac{k_{B} T d}{\pi c \hbar}\right]^{-1}$

$y \quad \hbar \omega /\left(k_{B} T\right)$

\section{Introduction}

Radiative transfer between two closely spaced objects can exhibit features not captured by the classical theory of radiative transfer [1 because of near-field phenomena, 
such as the collective influence of interference and diffraction effects and the tunneling of evanescent waves. One of the most striking features is the enhancement of radiative transfer beyond the blackbody limit due to tunneling of surface phonon polaritons [2, 3, 4, 5. This enhancement has been observed experimentally by measuring radiative transfer between a microsphere and a planar substrate [6. Though most of the recent studies on near-field enhancement have focused on the effect of surface phonon polaritons, what piqued the interest of researchers [7] in the mid-to-late 1960s was the enhancement of radiative transfer between metallic surfaces [8, 9, 10, 7, 11] at small gaps because of its importance in cryogenics. In this paper, we investigate the gap dependence of radiative transfer between two planar metallic surfaces. Unlike Polder and van Hove, who investigated this problem previously, [1] our focus is not on the enhancement due to evanescent waves but rather on an initial decrease in radiative transfer as the gap decreases ${ }^{1}$ In contrast to enhancement due to tunneling of evanescent waves, which becomes apparent at sub-micron gaps (usually $\lesssim 500 \mathrm{~nm}$ ), the minimum in radiative transfer occurs at larger gaps $(\approx 2 \mu \mathrm{m})[11$. Though evanescent waves contribute to radiative transfer at such gaps, as we will show, the decrease in radiative transfer can be explained by restricting ourselves to propagating waves. We realized in 2014, at NanoRad2014 (Second International Workshop on Nano-Micro Thermal Radiation) held in Shanghai, that Tsurimaki et al. 12 were also working on a similar problem. Tsurimaki et al. focus on the transition between the far-field regime and near-field regime between different types of materials (silicon carbide half-spaces and aluminum half-spaces). In this paper, we focus on radiative transfer between metallic half-spaces and the emphasis is on deriving analytical results.

The structure of the paper is as follows: In Sec. 2 we will describe briefly the theoretical basis for determining the radiative transfer between two identical half-spaces separated by a vacuum gap. In Sec. 3, we derive expressions for gap dependent radiative heat transfer coefficient due to propagating waves. Analytical expressions for radiative transfer at vacuum gaps beyond the value at which the minimum radiative transfer occurs are derived in Sec. 3.1 analytical expressions for vacuum gaps from contact to the minimum gap are derived in Sec. 3.2. In Sec. 4. analytical expressions for the minimum gap $d_{\text {min }}$ and the minimum heat transfer coefficient $h_{\min }$ are obtained, from which the dependence of $h_{\min }$ on the temperature and other optical properties of the metals can be extracted. The influence of evanescent waves and the possibility of measuring the decrease in radiative transfer are discussed.

\footnotetext{
${ }^{1}$ We got interested in this problem, in part, after a discussion on this topic with Prof. Pinar Menguc.
}

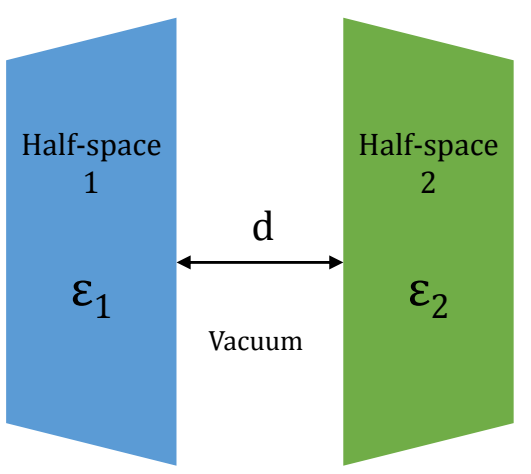

Figure 1: Two planar, metallic half-spaces are separated by a vacuum gap $d$. It is assumed in our case that the two half-spaces are silver $\left(\varepsilon_{1}=\varepsilon_{2}=\varepsilon_{A g}\right)$.

\section{Theoretical framework}

Even though we focus only on propagating waves, classical theory of radiative transfer is insufficient to capture interference effects due to multiple reflections of plane waves. Hence, we use Rytov's theory of fluctuational electrodynamics 13, 14 and the dyadic Green's function formalism to model heat flow between two half-spaces separated by a vacuum gap [15. Since the theory of fluctuational electrodynamics has been discussed extensively in many publications [15, 16, we use the well-known result for the linearized radiative heat transfer coefficient between two half-spaces of identical material separated by a vacuum gap of thickness $d$ (see Fig. 1):

$$
h_{p w}(d, T)=\frac{k_{B}^{4} T^{3}}{4 \pi^{2} c^{2} \hbar^{3}} \int_{0}^{\infty} d y \frac{y^{4} e^{y}}{\left(e^{y}-1\right)^{2}} \sum_{j=s, p} \int_{0}^{1} d x x \mathcal{T}_{j}(x, y)
$$

where $j=s, p$ refer to transverse electric and transverse magnetic polarizations respectively, $y=\hbar \omega / k_{B} T$ is a nondimensional frequency, $\omega$ is the angular frequency, $k_{B}$ is Boltzmann's constant, $2 \pi \hbar$ is Planck's constant, $T$ is the temperature of the two objects, $c$ is the speed of light in vacuum, $k_{0}=\frac{\omega}{c}, x=\frac{k_{z 0}}{k_{0}}$ is the non-dimensionalized $z$ wavevector in vacuum $\left(k_{z 0}\right.$ is the dimensional wavevector), $\mathcal{T}_{j}$ is the generalized transmissivity and is related to the polarization dependent Fresnel reflection coefficient, $R_{j}$, as:

$$
\mathcal{T}_{j}(x, y)=\frac{\left(1-\left|R_{j}\right|^{2}\right)^{2}}{\left|1-R_{j}^{2} e^{i 2 x k_{0} d}\right|^{2}}
$$

The subscript $p w$ for the heat transfer coefficient $h$ is used to indicate that only the contributions of propagating waves are taken into consideration. $R_{s}$ and $R_{p}$ are given by the following formulae:

$$
\begin{aligned}
& R_{s}=\frac{x-k_{z 1} / k_{0}}{x+k_{z 1} / k_{0}}=\frac{x-\sqrt{\varepsilon_{1}-1+x^{2}}}{x+\sqrt{\varepsilon_{1}-1+x^{2}}} \\
& R_{p}=\frac{k_{z 0}-k_{z 1} / \varepsilon_{1}}{k_{z 0}+k_{z 1} / \varepsilon_{1}}=\frac{x-\varepsilon_{1}^{-1} \sqrt{\varepsilon_{1}-1+x^{2}}}{x+\varepsilon_{1}^{-1} \sqrt{\varepsilon_{1}-1+x^{2}}}
\end{aligned}
$$


where $\varepsilon_{1}$ is the dielectric function of the half-spaces, and $k_{z 1}=k_{0} \sqrt{\varepsilon_{1}-1+x^{2}}$ is the $z$-wavevector in the halfspaces. Since the materials have to be highly reflective for all frequencies at which radiative transfer takes place, we take the material of the half-spaces to be metallic with the dielectric function described by the Drude model as follows:

$$
\varepsilon(\omega)=1-\frac{\omega_{p}^{2}}{\omega(\omega+i \gamma)}
$$

For purposes of simulations shown in this paper, we use the material properties of silver $\left(\omega_{p}=9.02 \mathrm{eV}, \gamma=0.018\right.$ eV) [17.

\section{Behavior of radiative transfer at different sepa- rations}

\subsection{Radiative transfer at medium to large gaps}

Since the distance dependence of $h_{p w}(d, T)$ arises from the integral $\int_{0}^{1} d x x \mathcal{T}_{j}(x)$, we study its behavior at different gaps in this section by paying specific attention to the denominator of $\mathcal{T}_{j}(x)$, i.e., $1-R_{j}^{2} e^{i 2 x k_{0} d}$. To show how different wavevectors contribute to the integral, the variation of $\left|1-R_{j}^{2} e^{i 2 x k_{0} d}\right|$ and $\left|1-R_{p}^{2} e^{i 2 x k_{0} d}\right|^{-2}$ for $\lambda=2 \pi / k_{0}=10$ $\mu \mathrm{m}, d=22 \mu \mathrm{m}$ are plotted in Fig. 2(a) and Fig. 2(b) respectively. The variation of $\left|1-R_{j}^{2} e^{i 2 x k_{0} d}\right|^{-2}$ shows that the integral over $x$ in Eq. 1 is dominated by the contribution from regions near the spikes (notice that the $y$-axis for Fig. 2(b) is in log-scale). For materials like silver, the reflection coefficients are relatively weak functions of $x$, which explains the fact that the spikes in Fig. 2 appear to be spaced equally apart. The spikes occur when the phase of $R_{j}^{2} e^{i 2 x k_{0} d}$, a plot of which is shown in Fig. 2(c), becomes an integral multiple of $2 \pi$. Expressed mathematically, it is:

$$
x k_{0} d+\arg \left(R_{j}\right)=n \pi
$$

At any wavelength (or frequency), let us denote by $x_{n}(n=1,2,3, \cdots)$ the locations of the local minima of $\left|1-R_{j}^{2} e^{i 2 x k_{0} d}\right|$. The values of $x_{n}$ are obtained by solving Eq. 5. Since the integral over $x$ in Eq. 1 is dominated by contributions from near $x_{n}$, we can approximate $\left|1-R_{j}^{2} e^{i 2 x k_{0} d}\right|$ as:

$$
\begin{aligned}
& \left.\left.\left|1-R_{j}^{2}(x) e^{i 2 x k_{0} d}\right|^{2} \approx|1-| R_{j}\left(x_{n}\right)\right|^{2} e^{i 2 k_{0} d\left(x-x_{n}\right)}\right|^{2} \\
& \quad \approx\left(1-\left|R_{j}\left(x_{n}\right)\right|^{2}\right)^{2}+4\left|R_{j}\left(x_{n}\right)\right|^{4} \sin ^{2}\left[k_{0} d\left(x-x_{n}\right)\right]^{2}
\end{aligned}
$$

In deriving the above expression, we have assumed that $R_{j}(x)$ does not change significantly in the region around $x=x_{n}$ and is approximated by its value at $x=x_{n}$. Using Eq. 6. $\mathcal{T}_{j}(x)$ can be approximated near $x=x_{n}$ as:

$$
\begin{aligned}
\mathcal{T}_{j}(x) & \approx\left[1+\frac{4\left|R_{j}\left(x_{n}\right)\right|^{4}}{\left(1-\left|R_{j}\left(x_{n}\right)\right|^{2}\right)^{2}} \sin ^{2}\left[k_{0} d\left(x-x_{n}\right)\right]\right]^{-1} \\
& \approx\left[1+\frac{4\left|R_{j}\left(x_{n}\right)\right|^{4}}{\left(1-\left|R_{j}\left(x_{n}\right)\right|^{2}\right)^{2}}\left(k_{0} d\right)^{2}\left(x-x_{n}\right)^{2}\right]^{-1}
\end{aligned}
$$
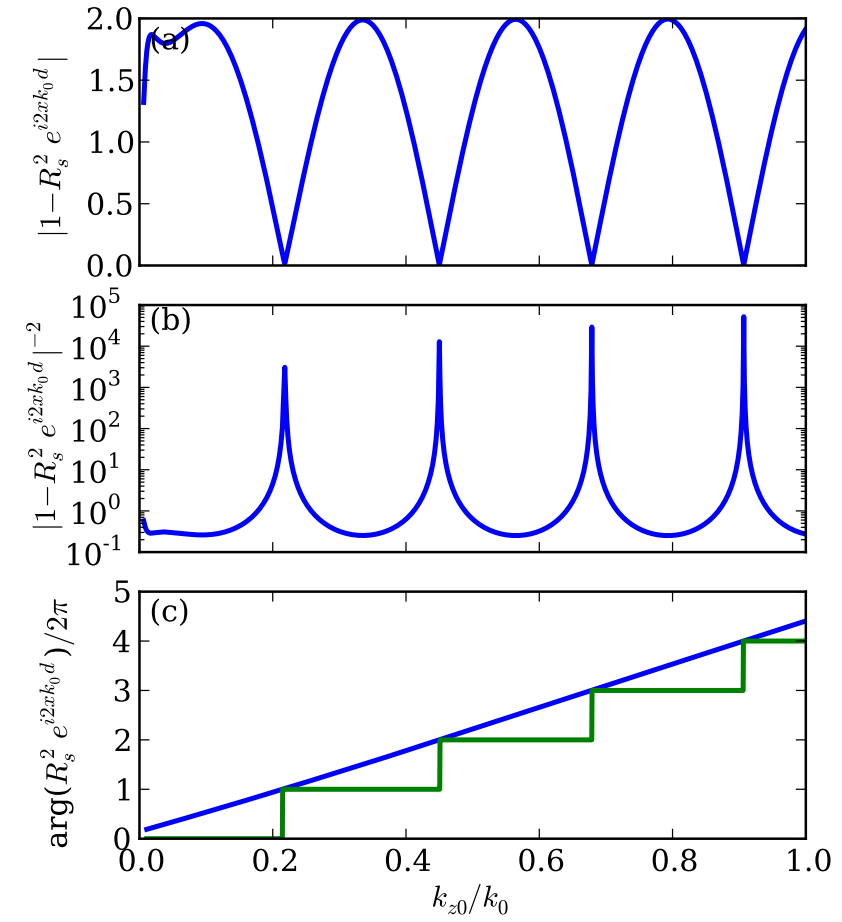

Figure 2: (Color online) Plots of $\left|1-R_{j}^{2} e^{i 2 x k_{0} d}\right|$ and $\mid 1-$ $\left.R_{j}^{2} e^{i 2 x k_{0} d}\right|^{-2}$ for $s$-polarization vs. $x$ at $\lambda=10 \mu \mathrm{m}$ for $d=22$ $\mu \mathrm{m}$ are shown in Fig. 2(a) and Fig. 2(b) respectively. The phase (blue line) of $R_{j}^{2} e^{i 2 x k_{0} d}$, in units of $2 \pi$, is shown in Fig. 22. c). The locations at which the phase crosses a multiple of $2 \pi$ are 1dentified (green line) to show that they coincide with the peaks.

Using Eq. 7. $\int_{0}^{1} d x x \mathcal{T}_{j}(x)$ (the finite limit over $x$ around each spike is replaced by $-\infty$ and $\infty$ because of the rapidly decreasing integrand) becomes

$$
\begin{aligned}
\int_{0}^{1} d x x \mathcal{T}_{j} & \approx \sum_{n} \int_{-\infty}^{\infty} d x \frac{x-x_{n}+x_{n}}{1+\frac{4\left|R_{j}\left(x_{n}\right)\right|^{4}\left(k_{0} d\right)^{2}}{\left(1-\left|R_{j}\left(x_{n}\right)\right|^{2}\right)^{2}}\left(x-x_{n}\right)^{2}} \\
& \approx \sum_{n} \frac{\pi x_{n}\left(1-\left|R_{j}\left(x_{n}\right)\right|^{2}\right)}{2 k_{0} d\left|R_{j}\left(x_{n}\right)\right|^{2}}
\end{aligned}
$$

By substituting Eq. 8 in Eq. 1, the gap-dependent radiative heat transfer coefficient can determined by evaluating the following integral:

$$
h_{p w}^{(l g)}(d, T) \approx \frac{15 \sigma T^{3}}{\pi^{4}} \int_{0}^{\infty} \frac{y^{4} e^{y} d y}{\left(e^{y}-1\right)^{2}} \sum_{\substack{n=0 \\ j=s, p}}^{m} \frac{\pi x_{n}\left(1-\left|R_{j}\left(x_{n}\right)\right|^{2}\right)}{2 k_{0} d\left|R_{j}\left(x_{n}\right)\right|^{2}}
$$

where $\sigma=\pi^{2} k_{B}^{4} / 60 c^{2} \hbar^{3}$ is the Stefan-Boltzmann constant. The superscript $l g$ refers to "large gap" since it does not capture the contribution from $x \approx 0$ when $k_{T} d \rightarrow 0$.

The expression for $h_{p w}^{(l g)}(d, T)$ in Eq. 9 can be further simplified if we can obtain simpler expressions for $1-\left|R_{j}\left(x_{n}\right)\right|^{2}$ and the values of $x_{n}$. The $\left|R_{j}\left(x_{n}\right)\right|^{2}$ term in the denominator of Eq. 9 can be replaced by unity because of the highly reflective nature of the metal-vacuum 
interface. The Fresnel reflection coefficients (Eq. 3) can be approximated as:

$$
R_{p}=\frac{\left(x n_{1}-1\right)+i x \kappa_{1}}{\left(x n_{1}+1\right)+i x \kappa_{1}} ; \quad R_{s}=\frac{\left(x-n_{1}\right)-i \kappa_{1}}{\left(x+n_{1}\right)+i \kappa_{1}}
$$

where it has been assumed that $k_{z 1} \approx k_{0}\left(n_{1}+i \kappa_{1}\right)$ because $n_{1}, \kappa_{1} \gg 1$ for the frequencies of interest $\left(\omega \ll \omega_{p}\right)$. By considering the behavior of $1-\left|R_{j}\left(x_{n}\right)\right|^{2}$ in the limit as $x \rightarrow 0$ and $x \rightarrow 1$, it is possible to show that the following expressions are good approximations for $1-\left|R_{j}\left(x_{n}\right)\right|^{2}$ :

$$
\begin{aligned}
1-\left|R_{p}^{a}(x)\right|^{2} & =\frac{4 n_{1} x}{\left(n_{1}^{2}+\kappa_{1}^{2}\right) x^{2}+1} \\
1-\left|R_{s}^{a}(x)\right|^{2} & =\frac{4 n_{1} x}{n_{1}^{2}+\kappa_{1}^{2}}
\end{aligned}
$$

The superscript "a" is used to indicate that $R_{j}^{a}(x) \approx$ $R_{j}(x)$. The actual and approximate values of $x\left(1-\left|R_{j}(x)\right|^{2}\right)$ are plotted in Fig. 3(a). The relative errors between the actual and approximate values of $x\left(1-\left|R_{j}(x)\right|^{2}\right)$ are shown in Fig. 3(b). For $x \gtrsim 0.1$, the relative error is less than $1 \%$ for both polarizations. By realizing that $\arg \left(R_{s}\right) \approx-\pi$ and $\arg \left(R_{p}\right) \approx 0$, we can see that $x_{n} \approx n \pi / k_{0} d$ with $n \geq 1$. Since $x_{n} \leq 1$, the maximum value of $n$ is $\left\lfloor\frac{k_{0} d}{\pi}\right\rfloor$, where the notation $\lfloor z\rfloor$ is used to represent the largest integer less than or equal to z. By using Eq. 11 and the approximate values of $x_{n}$, $\sum_{n} x_{n}\left(1-\left|R_{j}\left(x_{n}\right)\right|^{2}\right)$ can be approximated as

$$
\begin{gathered}
\sum_{n} x_{n}\left(1-\left|R_{p}^{a}\left(x_{n}\right)\right|^{2}\right)=\sum_{n} \frac{4 n_{1} x_{n}^{2}}{\left(n_{1}^{2}+\kappa_{1}^{2}\right) x_{n}^{2}+1} \\
\approx \sum_{n} \frac{4 n_{1}}{n_{1}^{2}+\kappa_{1}^{2}}=\frac{4 n_{1}}{n_{1}^{2}+\kappa_{1}^{2}}\left\lfloor\frac{k_{0} d}{\pi}\right\rfloor \\
\sum_{n} x_{n}\left(1-\left|R_{s}^{a}\left(x_{n}\right)\right|^{2}\right)=\frac{4 n_{1}}{n_{1}^{2}+\kappa_{1}^{2}} \sum_{n} x_{n}^{2} \\
=\frac{4 n_{1}}{n_{1}^{2}+\kappa_{1}^{2}} \frac{\left\lfloor\frac{k_{0} d}{\pi}\right\rfloor\left(\left\lfloor\frac{k_{0} d}{\pi}\right\rfloor+1\right)\left(2\left\lfloor\frac{k_{0} d}{\pi}\right\rfloor+1\right)}{6\left(k_{0} d / \pi\right)^{2}}
\end{gathered}
$$

Hence, we can obtain a further simplification for $h_{p w}^{(l g)}(d, T)$ which involves integration only over $y$ as:

$$
\begin{aligned}
h_{p w}^{(l g)}(d, T) \approx & \frac{15 \sigma T^{3}}{\pi^{4}} \int_{0}^{\infty} d y \frac{y^{4} e^{y}}{\left(e^{y}-1\right)^{2}} \frac{2 n_{1}}{n_{1}^{2}+\kappa_{1}^{2}} \frac{\left\lfloor\frac{k_{0} d}{\pi}\right\rfloor}{\frac{k_{0} d}{\pi}} \times \\
& \left(1+\frac{1}{6}\left[\frac{\left\lfloor\frac{k_{0} d}{\pi}\right\rfloor+1}{\frac{k_{0} d}{\pi}}\right]\left[\frac{2\left\lfloor\frac{k_{0} d}{\pi}\right\rfloor+1}{\frac{k_{0} d}{\pi}}\right]\right) \\
= & \frac{15 \sigma T^{3}}{\pi^{4}} \sum_{n=1}^{\infty} \int_{n \xi}^{(n+1) \xi} d y \frac{e^{y}}{\left(e^{y}-1\right)^{2}} \frac{2 n_{1}}{n_{1}^{2}+\kappa_{1}^{2}} \times \\
& \left(n \xi y^{3}+\frac{n(n+1)(2 n+1)}{6} \xi^{3} y\right)
\end{aligned}
$$
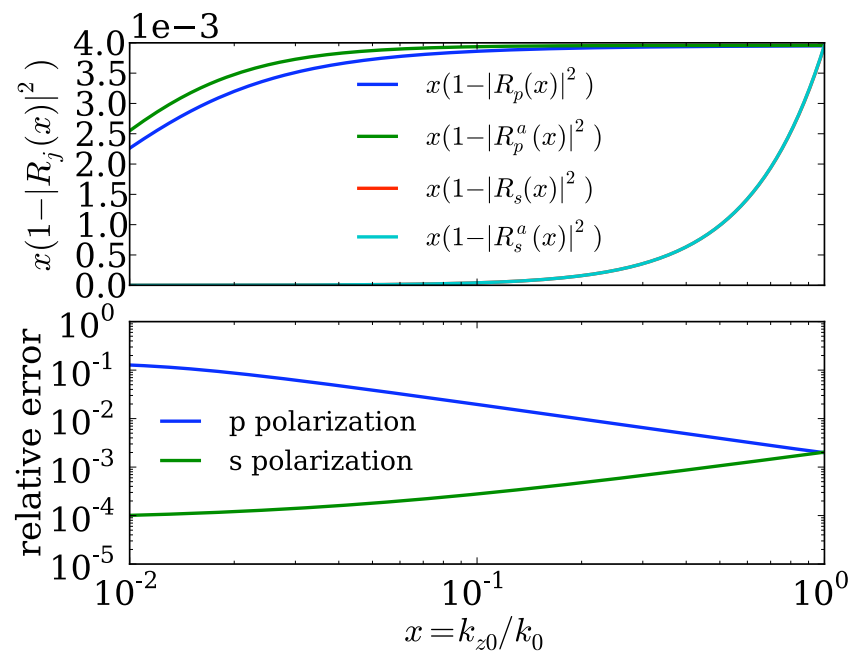

Figure 3: Approximations for $x\left(1-\left|R_{j}(x)\right|^{2}\right)$. The curves for $x(1-$ $\left.\left|R_{s}(x)\right|^{2}\right)$ and $x\left(1-\left|R_{s}^{a}(x)\right|^{2}\right)$ are almost coincident - hence only the $x\left(1-\left|R_{s}^{a}(x)\right|^{2}\right)$ is visible.

where $\xi=\frac{\pi c \hbar}{k_{B} T d}$. The physical interpretation of Eq. 13 is that as $d$ increases, a larger number of modes contribute to radiative transfer. When $d$ approaches $d_{\text {min }}$, only the $n=1$ term is important and an explicit expression for $h_{p w}(d, T)$ is given by:

$$
\begin{aligned}
h_{p w}^{(l g)}(d, T) & \approx \frac{15 \sigma T^{3}}{\pi^{4}} \int_{\xi}^{2 \xi} d y \frac{e^{y}}{\left(e^{y}-1\right)^{2}} \frac{2 n_{1}}{n_{1}^{2}+\kappa_{1}^{2}}\left(\xi y^{3}+\xi^{3} y\right) \\
& \approx \frac{\gamma}{\omega_{p}} \frac{15 \sigma T^{3}}{\pi^{4}} \xi \epsilon_{\gamma}^{4} \int_{\xi / \epsilon_{\gamma}}^{2 \xi / \epsilon_{\gamma}} d z z \sum_{n=1}^{\infty} n e^{-n \epsilon_{\gamma} z} \times \\
& \left(z^{2}+\frac{\xi^{2}}{\epsilon_{\gamma}^{2}}\right)\left[2 z\left(\sqrt{1+z^{2}}-z\right)\right]^{1 / 2}
\end{aligned}
$$

where $\epsilon_{\gamma}=\hbar \gamma / k_{B} T$ and $z=\omega / \gamma$. In going from line 1 to line 2 of Eq. 14, we have use the substitution $y=z \epsilon_{\gamma}$, and the approximate expression for $\frac{2 n_{1}}{n_{1}^{2}+\kappa_{1}^{2}}$ from Table 1 (useful expressions for deriving subsequent equations in this paper are also given in Table 1). In the limits $\xi / \epsilon_{\gamma} \gg 1$ and $\xi / \epsilon_{\gamma} \ll 1$, analytical expressions for approximate values of $h_{p w}^{(l g)}(d, T)$ near $d=d_{\min }$ can be determined as follows. When $\xi / \epsilon_{\gamma} \gg 1$, only those frequencies such that $z>\xi / \epsilon_{\gamma} \gg 1$ contribute to the radiative transfer. Hence, $\left[2 z\left(\sqrt{1+z^{2}}-z\right)\right]^{1 / 2}=\left[2 z^{2}\left(\sqrt{1+\frac{1}{z^{2}}}-1\right)\right]^{1 / 2} \approx 1$, 
and the expression for $h_{p w}^{(l g)}(d, T)$ can be written as:

$$
\begin{aligned}
h_{p w}^{(l g)}(d, T) & \approx \frac{\gamma}{\omega_{p}} \frac{15 \sigma T^{3}}{\pi^{4}} \xi \sum_{n=1}^{\infty} n \int_{\xi}^{2 \xi} d y e^{-n y}\left(y^{3}+y \xi^{2}\right) \\
& \approx \frac{\gamma}{\omega_{p}} \frac{15 \sigma T^{3}}{\pi^{4}} \xi \int_{\xi}^{\infty} d y\left(y^{3}+y \xi^{2}\right) e^{-y} \\
& =\frac{\gamma}{\omega_{p}} \frac{15 \sigma T^{3}}{\pi^{4}} e^{-\xi}\left(\xi^{4}+2 \xi^{3}+3 \xi^{2}+3 \xi\right)
\end{aligned}
$$

To derive Eq. 15 we have replaced the upper limit of the integral from $2 \xi$ to $\infty$ because of the large values of $\xi$ encountered near $d \approx d_{\min }$. It can be seen from Fig. 5 that $\xi \approx 10$ when $d=d_{\text {min }}$.

When $\xi \ll \epsilon_{\gamma}$, keeping in mind that $\xi \approx 10$ near $d=$ $d_{\text {min }}$, we can see that frequencies such that $z \ll 1$ dominate the contribution to the radiative transfer. Hence, $\left[2 z\left(\sqrt{1+z^{2}}-z\right)\right]^{1 / 2} \approx \sqrt{2 z}$, and $h_{p w}^{(l g)}(d, T)$ can be written as:

$$
\begin{array}{r}
h_{p w}^{(l g)}(d, T) \approx\left[\frac{\gamma}{\omega_{p}} \frac{30 \sigma T^{3}}{\pi^{4}}\right] \times \\
\quad \sqrt{\frac{\xi^{2}}{2 \epsilon_{\gamma}} \sum_{n=1}^{\infty} n \int_{\xi}^{\infty} d y\left(y^{7 / 2}+\frac{\xi^{2}}{\epsilon_{\gamma}^{2}} y^{3 / 2}\right) e^{-n y}} \\
\approx \sqrt{\frac{\xi^{2}}{2 \epsilon_{\gamma}}} \sum_{n=1}^{\infty}\left[\begin{array}{c}
\frac{e^{-n \xi} \sqrt{\xi}}{8}\left(\frac{105}{n^{3}}+\frac{70 \xi}{n^{2}}+\frac{40 \xi^{2}}{n}+16 \xi^{3}\right) \\
+\frac{\left(105+12 n^{2} \xi^{2}\right)}{16 n^{7 / 2}} \sqrt{\pi} \operatorname{erfc}(\sqrt{n \xi})
\end{array}\right] \\
\approx \sqrt{\frac{2 \xi}{\epsilon_{\gamma}}}\left(\xi^{4}+\frac{5 \xi^{3}}{2}+\frac{19 \xi^{2}}{4}+\frac{105 \xi}{16}+\frac{105}{32}\right) e^{-\xi}
\end{array}
$$

In summary, when $d \approx d_{\text {min }}, h_{p w}^{(l g)}(d, T)$ can be written as:

$$
h_{p w}^{(l g)}(d, T) \approx \frac{\gamma}{\omega_{p}} \frac{30 \sigma T^{3}}{\pi^{4}} f_{l g}\left(\xi, \epsilon_{\gamma}\right) e^{-\xi},
$$

where

$f_{l g}\left(\xi, \epsilon_{\gamma}\right)= \begin{cases}\xi^{4}+2 \xi^{3}+3 \xi^{2}+3 \xi, & \left(\epsilon_{\gamma} \ll \xi\right) \\ \sqrt{\frac{2 \xi}{\epsilon_{\gamma}}}\left(\xi^{4}+\frac{5}{2} \xi^{3}+\frac{19}{4} \xi^{2}+\frac{105}{16} \xi+\frac{105}{32}\right), & \left(\epsilon_{\gamma} \gg \xi\right)\end{cases}$

\subsection{Radiative transfer at small to medium gaps}

The spikes seen in $\mathcal{T}_{j}(x)$ at larger gaps vanish for $\arg \left(R_{j}^{2} e^{i 2 x k_{0} d}\right)$ less than $2 \pi$ (similar to the case when $k_{0} d<\pi$ when the two half spaces are perfect electric conductors). Because of the finite value of $\gamma$, even frequencies satisfying $k_{0} d<\pi$ contribute to energy transfer. When $k_{0} d \ll 1$ (note that $k_{0} d \ll 1$ but $k_{p} d=\omega_{p} d / c$ need not be small), the following expressions can be obtained by using Eq. 10 .

$$
\begin{aligned}
1-R_{p}^{2} e^{i 2 k_{0} d x} & =e^{i k_{0} d x}\left(e^{-i k_{0} d x}-R_{p}^{2} e^{i k_{0} d x}\right) \\
& \approx \frac{4 x\left(n_{1}+i \kappa_{1}\right)\left[1-i \frac{k_{0} d}{2} x^{2}\left(n_{1}+i \kappa_{1}\right)\right]}{\left[x\left(n_{1}+i \kappa_{1}\right)+1\right]^{2} e^{-i k_{0} d x}}
\end{aligned}
$$

Table 1: Various useful approximations valid when $\omega \ll \omega_{p}(z=$ $\omega / \gamma)$

\begin{tabular}{ll}
\hline \hline Entity & Expression \\
\hline$k_{0}^{2} d^{2}\left(n_{1}^{2}+\kappa_{1}^{2}\right)$ & $\frac{\left(k_{p} d\right)^{2} z}{\sqrt{1+z^{2}}}$ \\
$n_{1} k_{0} d$ & $k_{p} d \sqrt{\frac{z}{\sqrt{1+z^{2}}}}\left(\sqrt{1+z^{2}}-z\right)^{1 / 2}$ \\
$\frac{n_{1}^{2}}{n_{1}^{2}+\kappa_{1}^{2}} \frac{1}{n_{1} k_{0} d}$ & $\frac{1}{k_{p} d} \sqrt{\frac{1}{2 z}}\left(\sqrt{1+z^{2}}-z\right)^{1 / 2}$ \\
$\frac{n_{1} k_{0} d}{2+\kappa_{1} k_{0} d}$ & $\frac{k_{p} d \sqrt{z\left(\sqrt{1+z^{2}}-z\right)}}{2 \sqrt{2\left(1+z^{2}\right)}+k_{p} d \sqrt{z\left(\sqrt{1+z^{2}}+z\right)}}$ \\
$\frac{4 n_{1}}{n_{1}^{2}+\kappa_{1}^{2}}$ & $\frac{\gamma}{\omega_{p}}\left[8 z\left(\sqrt{1+z^{2}}-z\right)\right]^{1 / 2}$ \\
\hline
\end{tabular}

$$
\begin{aligned}
1-R_{s}^{2} e^{i 2 k_{0} d x} & =e^{i k_{0} d x}\left(e^{-i k_{0} d x}-R_{s}^{2} e^{i k_{0} d x}\right) \\
& \approx \frac{4 x\left(n_{1}+i \kappa_{1}\right)\left[1-i \frac{k_{0} d}{2}\left(n_{1}+i \kappa_{1}\right)\right]}{\left(x+n_{1}+i \kappa_{1}\right)^{2} e^{-i k_{0} d x}}
\end{aligned}
$$

By substituting Eq. 18, Eq. 19, and Eq. 11 in Eq. 2 we obtain the following expressions for the polarization dependent generalized transmissivity:

$$
\begin{aligned}
& \mathcal{T}_{p}(x)=\frac{\left(\frac{n_{1}^{2}}{n_{1}^{2}+\kappa_{1}^{2}}\right)}{\left[1+\kappa_{1} k_{0} d x^{2}+\left(\frac{k_{0} d}{2}\right)^{2}\left(n_{1}^{2}+\kappa_{1}^{2}\right) x^{4}\right]} \\
& \mathcal{T}_{s}(x)=\frac{\frac{n_{1}^{2}}{n_{1}^{2}+\kappa_{1}^{2}}}{\left[1+\kappa_{1} k_{0} d+\left(\frac{k_{0} d}{2}\right)^{2}\left(n_{1}^{2}+\kappa_{1}^{2}\right)\right]}
\end{aligned}
$$

Hence $\int_{0}^{1} d x x \mathcal{T}_{j}(x)(j=s, p)$ can be written as:

$$
\begin{aligned}
& \int_{0}^{1} d x x \mathcal{T}_{p}(x) \approx \frac{n_{1}^{2}}{n_{1}^{2}+\kappa_{1}^{2}} \frac{1}{n_{1} k_{0} d} \tan ^{-1}\left(\frac{n_{1} k_{0} d}{2+\kappa_{1} k_{0} d}\right) \\
& \int_{0}^{1} d x x \mathcal{T}_{s}(x) \approx \frac{\left(\frac{n_{1}^{2}}{2\left(n_{1}^{2}+\kappa_{1}^{2}\right)}\right)}{\left[1+\kappa_{1} k_{0} d+\left(\frac{k_{0} d}{2}\right)^{2}\left(n_{1}^{2}+\kappa_{1}^{2}\right)\right]}
\end{aligned}
$$

From Eqs. 22 and 23 the average transmissivity $\left(\int_{0}^{1} d x x \mathcal{T}_{p}(x) / \int_{0}^{1} d x x\right)$ at $d=0$ is $n_{1}^{2} /\left(n_{1}^{2}+\kappa_{1}^{2}\right)$ for both polarizations. Though the average transmissivities at larger gaps are not identical, the polarization becomes unimportant as $k_{p} d \rightarrow 0$ since the two half-spaces are effectively like a homogeneous medium. On the other hand, despite the two half-spaces almost merging into one, the transmissivity is not unity. The reason for this apparent discrepancy is that the generalized transmissivity is 
not just the transmissivity of a plane wave incident on a thin film. The transmissivity of a plane wave incident at a metal-vacuum interface to the second half-space is given by $\left(1-R_{j}^{2}\right) e^{i x k_{0} d} /\left(1-R_{j}^{2} e^{i 2 x k_{0} d}\right)$, which does indeed approach unity as $k_{0} d \rightarrow 0$. The transmissivity, as defined in Eq. 2 . also takes into account the attenuation of waves, generated by thermally induced fluctuations of charges distributed throughout the half-spaces, as they propagate through the half-space. If the materials were non-absorbing $\left(\kappa_{1} \ll n_{1}\right)$, then the average transmissivity $n_{1}^{2} /\left(n_{1}^{2}+\kappa_{1}^{2}\right)$ would indeed approach unity.

The heat transfer coefficient due to the low frequency modes, $h_{p w}^{(s g)}(d, T)$, can be written as:

$$
h_{p w}^{(s g)}(d, T)=\frac{\gamma}{\omega_{p}} \frac{30 \sigma T^{3}}{\pi^{4}} f_{s g}\left(\epsilon_{\gamma}, \xi, \epsilon_{\omega_{p}}\right)
$$

where $f_{s g}\left(\epsilon_{\gamma}, \xi, \epsilon_{\omega_{p}}\right)=\frac{1}{2} \int_{0}^{\infty} d y \frac{y^{4} e^{y}}{\left(e^{y}-1\right)^{2}} \int_{0}^{1} d x x\left[\mathcal{T}_{p}(x)+\mathcal{T}_{s}(x)\right]$ can be evaluated numerically using Eqs. 22 and 23 . Since $\omega_{p} \gg \gamma, f_{s g}\left(\epsilon_{\gamma}, \xi, \epsilon_{\omega_{p}}\right)$ is only a weak function of $\epsilon_{\omega_{p}}$. It can be seen from Eq. 22 and Eq. 23, and the appropriate expressions from Table 1 , that $\int_{0}^{1} d x x \mathcal{T}_{p}(x) \propto\left(k_{p} d\right)^{-1}$ whereas $\int_{0}^{1} d x x \mathcal{T}_{s}(x) \propto\left(k_{p} d\right)^{-2}$. To obtain analytical expressions for $f_{s g}\left(\epsilon_{\gamma}, \xi, \epsilon_{\omega_{p}}\right)$ near $d=d_{\text {min }}$, we neglect the $s$-polarization contribution to arrive at the following form:

$$
\begin{array}{r}
f_{s g}=\int_{0}^{\infty} d y \frac{y^{4} e^{y}}{\left(e^{y}-1\right)^{2}} \frac{n_{1}^{2}}{n_{1}^{2}+\kappa_{1}^{2}} \frac{1}{2 n_{1} k_{0} d} \tan ^{-1}\left(\frac{n_{1} k_{0} d}{2+\kappa_{1} k_{0} d}\right) \\
=\frac{\epsilon_{\gamma}^{4} \xi}{2 \pi} \int_{0}^{\infty} d z \frac{z^{4} e^{-\epsilon_{\gamma} z}}{\left(1-e^{-\epsilon_{\gamma} z}\right)^{2}} \frac{\left(\sqrt{1+z^{2}}-z\right)^{1 / 2}}{\sqrt{2 z}} \times \\
\tan ^{-1}\left[\frac{k_{p} d \sqrt{z\left(\sqrt{1+z^{2}}-z\right)}}{\sqrt{8\left(1+z^{2}\right)}+k_{p} d \sqrt{z\left(\sqrt{1+z^{2}}+z\right)}}\right]
\end{array}
$$

As in the case of $f_{l g}$, the two limits to consider are $\epsilon_{\gamma} \gg 1$ and $\epsilon_{\gamma} \ll 1$. The presence of the $e^{-\epsilon_{\gamma} z}$ term dictates that for $\epsilon_{\gamma} \gg 1$ frequencies such that $z \ll 1$ contribute most to $f_{s g}$. When $z \ll 1$ and $k_{p} d \gg 1\left(k_{p} d \approx 90\right.$ for $\omega_{p}=9 \mathrm{eV}$ and $d=2 \mu \mathrm{m})$, the $\tan ^{-1}$ term is replaced by $\pi / 4$ so that

$$
\begin{aligned}
f_{s g} & =\frac{\epsilon_{\gamma}^{4} \xi}{2 \pi} \int_{0}^{\infty} d z \frac{z^{4} e^{-\epsilon_{\gamma} z}}{\left(1-e^{-\epsilon_{\gamma} z}\right)^{2}} \frac{1}{\sqrt{2 z}} \times \frac{\pi}{4} \\
& =\frac{\xi}{8} \int_{0}^{\infty} d y \frac{y^{3} e^{-y}}{\left(1-e^{-y}\right)^{2}} \sqrt{\frac{y}{2 \epsilon_{\gamma}}} \\
& =\frac{\xi}{\sqrt{\epsilon_{\gamma}}} \frac{13.1059}{8 \sqrt{2}}=1.1584 \frac{\xi}{\sqrt{\epsilon_{\gamma}}}
\end{aligned}
$$

When $\epsilon_{\gamma} \ll 1$, frequencies such that $z>1$ contribute most to $f_{s g}$. Under this condition, we can neglect $\sqrt{8\left(1+z^{2}\right)}$ in the denominator of the $\tan ^{-1}$ term so that

$$
\begin{aligned}
& f_{s g} \approx \frac{\epsilon_{\gamma}^{4} \xi}{2 \pi} \int_{0}^{\infty} d z \frac{z^{4} e^{-\epsilon_{\gamma} z}}{\left(1-e^{-\epsilon_{\gamma} z}\right)^{2}} \frac{1}{\sqrt{2}} \sqrt{\frac{1}{2 z^{2}}} \times \\
& \tan ^{-1}\left[\sqrt{\frac{\sqrt{1+z^{2}}-z}{\sqrt{1+z^{2}}+z}}\right] \\
& \approx \frac{\epsilon_{\gamma}^{4} \xi}{2 \pi} \frac{1}{4} \int_{0}^{\infty} d z \frac{z^{2} e^{-\epsilon_{\gamma} z}}{\left(1-e^{-\epsilon_{\gamma} z}\right)^{2}}=\frac{\xi \epsilon_{\gamma}}{8 \pi} \int_{0}^{\infty} d y \frac{y^{2} e^{-y}}{\left(1-e^{-y}\right)^{2}} \\
&=\frac{\pi \xi \epsilon_{\gamma}}{24}
\end{aligned}
$$

In summary, when $d \approx d_{m i n}, f_{s g}\left(\epsilon_{\gamma}, \xi, \epsilon_{\omega_{p}}\right)$ can be written as:

$$
f_{s g}\left(\epsilon_{\gamma}, \xi, \epsilon_{\omega_{p}}\right)= \begin{cases}\pi \xi \epsilon_{\gamma} / 24, & \epsilon_{\gamma} \ll 1 \\ 1.1584 \xi / \sqrt{\epsilon_{\gamma}}, & \epsilon_{\gamma} \gg 1\end{cases}
$$

\section{Discussion}

We plot in Fig. 4 the radiative heat transfer coefficient computed by two different methods: (1) exact numerical evaluation of Eq. 1. and (2) approximate calculations using Eqs. 13 and 24 It can be seen that the exact and approximate calculations are in good agreement with each other. In the inset to Fig. 4 we also show the spectral contribution to the radiative transfer coefficient at different gaps. It can be seen from these insets that as the gap decreases, lower frequencies contribute more to radiative transfer. In particular, at $d=1.75 \mu \mathrm{m}$, one can notice contributions from the low frequency spectrum as well as the $n=1$ mode. Further details on the spectrum of energy transfer due to propagating waves can be found in Polder and van Hove [11.

The total heat transfer coefficient at any gap near $d_{\text {min }}$ is given by:

$$
h_{p w}(d, T)=\frac{30 \sigma T^{3}}{\pi^{4}} \frac{\gamma}{\omega_{p}}\left[f_{l g}\left(\xi, \epsilon_{\gamma}\right) e^{-\xi}+f_{s g}\left(\epsilon_{\gamma}, \xi, \epsilon_{\omega_{p}}\right)\right]
$$

with $f_{l g}$ and $f_{s g}$ given by Eqs. $17 \mathrm{~b}$ and 28 . From computed values of $h_{p w}(d, T)$ for $\gamma$ ranging from $0.001 \mathrm{eV}$ to $1 \mathrm{eV}, T=50,100,200,300 \mathrm{~K}$, and $\omega_{p}=10 \mathrm{eV}$, the non-dimensional gap $\xi_{\text {min }}^{-1}$ at which the minimum in radiative transfer occurs is determined numerically (keep in mind that $\omega_{p}$ does not have a strong influence on $d_{\text {min }}$ or $\left.\xi_{\text {min }}\right)$. The dependence of the resulting values of $\xi_{\text {min }}^{-1}$ on $\epsilon_{\gamma}$ is shown in Fig. 5 a. In Fig. 5b, the nondimensional minimum radiative heat transfer coefficient, $h_{\min }\left[\frac{30 \sigma T^{3}}{\pi^{4}} \frac{\gamma}{\omega_{p}}\right]^{-1}$, is plotted.

Though obtaining a functional relation between $\xi_{\text {min }}$ and $\epsilon_{\gamma}$ for all values of $\epsilon_{\gamma}$ maybe unlikely, expressions in the $\epsilon_{\gamma} \ll \xi_{\min }$ and $\epsilon_{\gamma} \gg \xi_{\min }$ limits can be determined. 


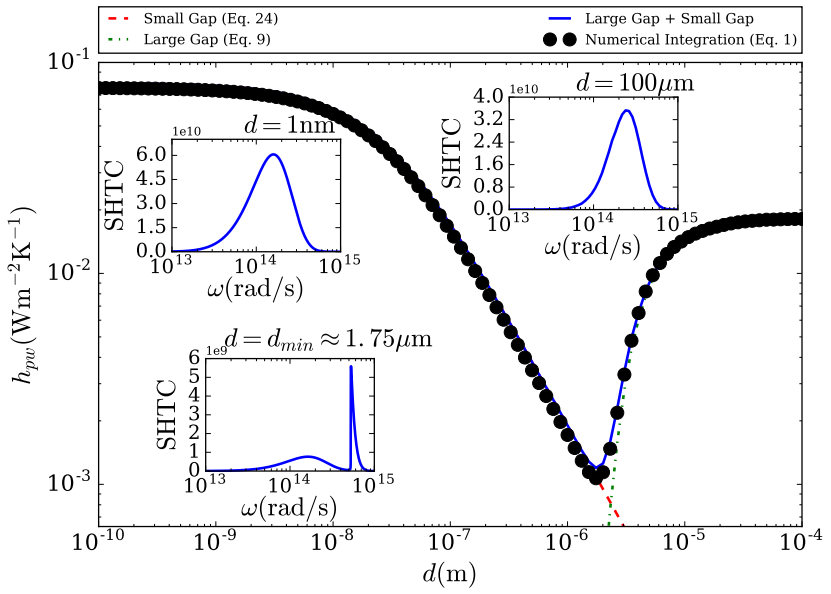

Figure 4: Variation of linearized radiative heat transfer coefficient with gap. Circular markers - numerical integration of Eq. 1 Dashed line - Eq. 9 Dashed-dotted - Eq. 24 Solid - Eq. $9+$ Eq. 24 Spectral contributions to $h_{p w}$ at different gaps $d(d=1 \mathrm{~nm}$ - near field, $d=1.75 \mu \mathrm{m}$ - near $d_{m i n}$, and $d=100 \mu \mathrm{m}$ - far field) are also shown in the inset. SHTC refers to spectral heat transfer coefficient in units of $\mathrm{Wm}^{-2} \mathrm{~K}^{-1}(\mathrm{rad} / \mathrm{s})^{-1}$. Since the $x$-axis of the spectral distributions are logarithmic, the $y$-axis has been scaled suitably to ensure that the area under the curves is indeed the radiative heat transfer coefficient. All computations are done at $T=315 \mathrm{~K}$.
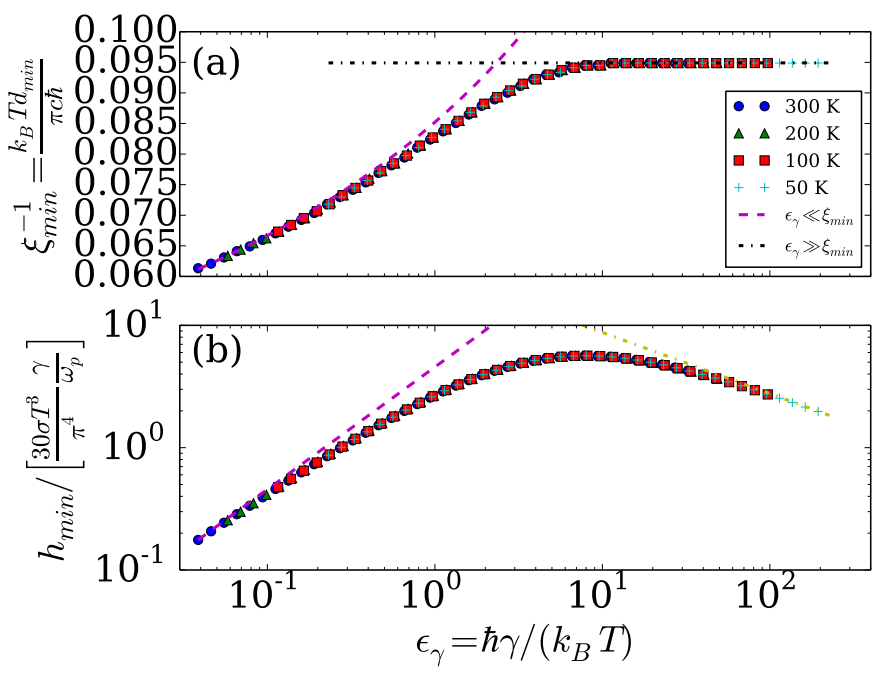

Figure 5: Plots of non-dimensional $\xi_{m i n}^{-1}$ (Fig. 5a) and nondimensionalized $h_{\min }$ (Fig. 5 5 ). The legend is the same for both sub-figures. The dashed and dashed-dotted lines correspond, respectively, to the $\varepsilon_{\gamma} \ll 1 \ll \xi_{\min }$ and $\varepsilon_{\gamma} \gg \xi_{\min } \gg 1$ limits of Eq. 32 (Fig. 5a) and Eq. 33 (Fig. 5p). $\xi_{\min }$ is obtained by solving the following implicit equation:

$$
\left[f_{l g}\left(\xi_{m i n}, \epsilon_{\gamma}\right)-f_{l g}^{\prime}\left(\xi_{m i n}, \epsilon_{\gamma}\right)\right] e^{-\xi_{m i n}}=f_{s g}^{\prime}\left(\epsilon_{\gamma}, \xi_{m i n}, \epsilon_{\omega_{p}}\right)
$$

where $f_{l g}^{\prime}$ and $f_{s g}^{\prime}$ refer to derivative of $f_{l g}$ and $f_{s g}$, respectively, with respect to $\xi$. Since Eq. 30 is transcendental, we further simplify the equation by approximating $f_{l g}\left(\xi_{m i n}, \epsilon_{\gamma}\right)-f_{l g}^{\prime}\left(\xi_{m i n}, \epsilon_{\gamma}\right)$, over a range of $\xi$ that contains $\xi_{\text {min }}$, with a function of the form $e^{a+b \xi_{\text {min }}}$. When $\epsilon_{\gamma} \ll \xi_{\text {min }}, f_{l g}-f_{l g}^{\prime} \approx e^{a+b \xi}\left(f_{l g}\right.$ defined in Eq. $17 \mathrm{~b}$ ), where $a=6.2446, b=0.2946$. The values of $a$ and $b$ are obtained by least squares fitting over the region $10 \leq \xi \leq 16.7$. We obtain the following solution for $\xi_{\text {min }}$ :

$$
\xi_{\text {min }} \approx \frac{a-\ln f_{s g}^{\prime}\left(\epsilon_{\gamma}, \xi_{\text {min }}, \epsilon_{\omega_{p}}\right)}{1-b} \approx 8.853-1.418 \ln \left[\frac{\pi}{24} \epsilon_{\gamma}\right]
$$

When $\epsilon_{\gamma} \gg \xi_{\text {min }}, f_{l g}-f_{l g}^{\prime} \approx \sqrt{\frac{2}{\epsilon_{\gamma}}} e^{a+b \xi}$, where $a=$ $5.22665, b=0.48502$ (over the region $9.5 \leq \xi \leq 10.6$ ). Since $f_{s g}=1.1584 \xi / \sqrt{\epsilon_{\gamma}}$ when $\epsilon_{\gamma} \gg \xi_{\text {min }}, \xi_{\text {min }} \approx 10.536$ in this limit. Because both $f_{l g}, f_{s g} \propto \epsilon_{\gamma}^{-1 / 2}, \xi_{\text {min }}$ turns out to be independent of $\epsilon_{\gamma}$ in this limit (as seen from Fig. 5a). To summarize,

$$
\xi_{\text {min }} \approx \begin{cases}8.853-1.418 \ln \left[\frac{\pi}{24} \epsilon_{\gamma}\right] & \text { if } \epsilon_{\gamma} \ll 1 \ll \xi_{\text {min }} \\ 10.536 & \text { if } \epsilon_{\gamma} \gg \xi_{\text {min }} \gg 1\end{cases}
$$

The dependence of $h_{\min }$ on other parameters, especially temperature, can be determined by substituting Eq. 32 in Eq. 29. When $\epsilon_{\gamma} \ll 1 \ll \xi_{m i n}, f_{l g} \approx \xi_{\text {min }}^{4}-$ only the leading order term in the polynomial expression for $f_{l g}$ is retained since $\xi_{\text {min }} \gtrsim 10.0$. By including the next order term, we obtain a more accurate approximation, given by

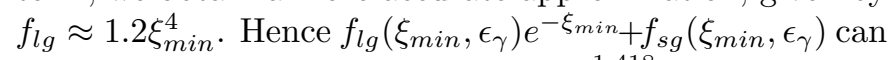
be shown to be $1.2 \xi_{\min }^{4} e^{-8.853}\left(\frac{\pi}{24} \epsilon_{\gamma}\right)^{1.418}+\xi_{\min }\left(\frac{\pi}{24} \epsilon_{\gamma}\right)$. Since $\xi_{\text {min }} \gtrsim 10$, and $\epsilon_{\gamma} \ll 1$, the asymptotic behavior in this regime is given by $\xi_{\min }\left(\frac{\pi}{24} \epsilon_{\gamma}\right)$. When $\epsilon_{\gamma} \gg \xi_{\min } \gg 1$, $\xi_{\text {min }}=10.536$ and $f_{l g}\left(\xi_{m i n}, \epsilon_{\gamma}\right) e^{-\xi_{m i n}}+f_{s g}\left(\xi_{m i n}, \epsilon_{\gamma}\right) \approx$ $\frac{7.2}{\sqrt{\epsilon_{\gamma}}}$. Using the results obtained above, the asymptotic behavior of $h_{\min }$ is given by:

$$
h_{\text {min }} \approx \begin{cases}\frac{30 \sigma T^{3}}{\pi^{4}} \frac{\gamma}{\omega_{p}} \frac{\pi \xi_{m i n} \epsilon_{\gamma}}{24} & \sim T^{2} \text { if } \epsilon_{\gamma} \ll 1 \ll \xi_{m i n} \\ \frac{30 \sigma T^{3}}{\pi^{4}} \frac{\gamma}{\omega_{p}} \frac{7.2}{\sqrt{\epsilon_{\gamma}}} & \sim T^{7 / 2} \text { if } \epsilon_{\gamma} \gg \xi_{m i n} \gg 1\end{cases}
$$

From Eq. 33 it follows that $h_{\min } \propto T^{2}$ in the high temperature limit and $h_{\min } \propto T^{7 / 2}$ in the low temperature limit.

As interesting as the phenomena of reduction in radiative transfer is, is it something that can be observed in an experiment? To answer that, we calculated the total radiative transfer (including the evanescent wave contribution) since it is the total radiative transfer, $h_{t o t}$, that is measured in any heat transfer experiment. The total radiative 


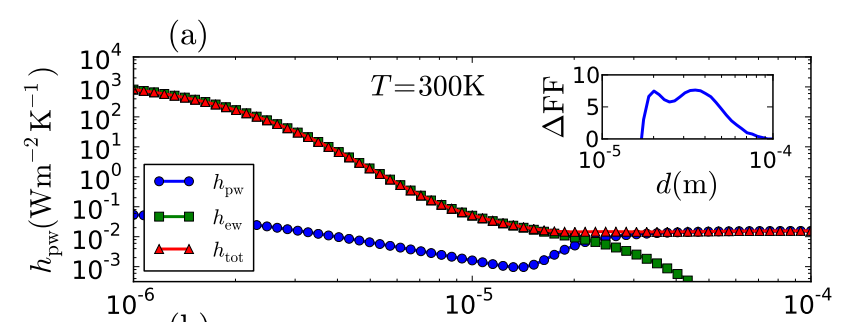

(b)

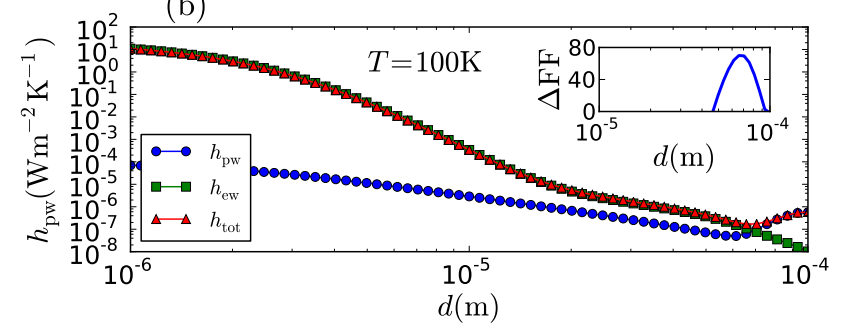

Figure 6: The propagating, evanescent, and total heat transfer coefficients ( $h_{\mathrm{pw}}, h_{\mathrm{ew}}$, and $h_{\mathrm{tot}}$, respectively) plotted vs. gap, $d$.

energy transfer between the two half-spaces is plotted as a function of the spacing $d$ and temperature $T$ in Fig. 6. The inset plots in Fig. 6] show $\Delta F F=100 \times\left(h_{f f}-h_{t o t}\right) / h_{f f}$, the percent change in $h_{t o t}$ relative to the far-field heat transfer coefficient $h_{f f}\left(h_{p w}(d, T)\right.$ as $\left.d \rightarrow \infty\right) . \Delta F F$ has been defined such that it is positive in regions of interest, where the heat transfer coefficient has decreased relative to that of the far-field. Clearly, we can see that the evanescent wave contribution at $300 \mathrm{~K}$ (Fig. 6a) is sufficiently large that the sharp minimum seen in Fig. 4 is now absent. However, we can alter the temperature and see that at some temperatures (radiative transfer at $100 \mathrm{~K}$ is shown in Fig. 6b) the evanescent wave contribution is not large enough to mask the suppression of radiative transfer, and $d_{\text {min }}$ is shifted to a higher value. For silver, we notice that at cryogenic temperatures, the percent decrease in the total heat transfer coefficient relative to the far-field value is approximately one order of magnitude higher than that corresponding to $T=300 \mathrm{~K}$. By exploiting this behavior, the minimum could be rendered observable, though we anticipate the experiment to be quite challenging. Two recent experimental investigations of near-field radiative transfer between metallic parallel surfaces have failed to notice the depression in radiative heat transfer as the gap is reduced before the eventual increase due to evanescent waves [18, 19]. See Tsurimaki et al. [12] for a more indepth discussion of the influence of evanescent waves on the extent of decrease in radiative heat transfer coefficient.

Another possibility of observing $h_{\min }$ could be to design metamaterials (metallo-dielectric multilayer films or nanocomposites, or by $1 \mathrm{D} / 2 \mathrm{D}$ surface texturing) which suppress the enhancement due to evanescent waves while preserving the multiple reflections that give rise to the nonmonotonic variation of the radiative heat transfer coefficient. This goal is opposite to what most works published so far in literature wish to achieve, namely that of increasing radiative transfer through evanescent or surface wave contributions. Because the suppression is observable at cryogenic temperatures, this phenomenon could be exploited to reduce heat loss through radiative transfer in cryogenic devices. However, a simpler technique of reducing radiative transfer is to use radiation shields [20].

\section{Conclusion}

Multiple reflections of propagating waves between two identical metallic half-spaces gives rise to a non-monotonic variation of radiative heat transfer coefficient with gap. The decrease in the magnitude of the minimum radiative heat transfer coefficient, compared to the far-field value, is dependent on how reflective the vacuum/half-space interface is. The minimum occurs because of a balance between lower energy transfer due to decreasing number of modes between the half-spaces as the gap decreases, and higher energy transfer due to low frequency contributions that decay to zero at larger gaps. The non-dimensional gap $\xi_{\text {min }}$ varies logarithmically with $\hbar \gamma / k_{B} T$, which explains why $d_{\min }$ is closely spaced for most of the metals under consideration here. We show that $h_{\text {min }} \propto T^{2}$ in the high temperature limit and $h_{\min } \propto T^{7 / 2}$ in the low temperature limit. Finally, we have proposed an experiment in which this effect may be observable at cryogenic temperatures.

This work was funded partially by ONR Grant N0001412-1-0996 and NSF IGERT DGE-1069240.

\section{References}

[1] Planck, M.. The Theory of Heat Radiation. New York: Dover Publications; 1991.

[2] Mulet, J.P., Joulain, K., Carminati, R., Greffet, J.J.. Enhanced Radiative Transfer at Nanometric Distances. Microscale Thermophys Eng 2002;6:209-222.

[3] Francoeur, M., Menguc, M., Vaillon, R.. Near-field radiative heat transfer enhancement via surface phonon polaritons coupling in thin films. Appl Phys Lett 2008;93(4):043109-043109.

[4] Francoeur, M., Pinar Menguc, M.. Role of fluctuational electrodynamics in near-field radiative heat transfer. J Quant Spectrosc Radiat Transfer 2008;109(2):280-293.

[5] Narayanaswamy, A., Chen, G.. Surface Modes for Near Field Thermophotovoltaics. apl 2003;82:3544-3546.

[6] Shen, S., Narayanaswamy, A., Chen, G.. Surface Phonon Polaritons Mediated Energy Transfer between Nanoscale Gaps. Nano Lett 2009;9:2909-2913.

[7] Domoto, G.A., Tien, C.L.. Thick Film Analysis of Radiative Transfer Between Parallel Metallic Surfaces. jhttasme 1970;92:399-404.

[8] Cravalho, E.G., Tien, C.L., Caren, R.P.. Effect of Small Spacings on Radiative Transfer Between Two Dielectrics. jhttasme 1967;89:351-358.

[9] Hargreaves, C.. Anomalous radiative transfer between closelyspaced bodies. Phys Lett A 1969;30a:491 - 2 .

[10] Hargreaves, C.. Radiative transfer between closely spaced bodies. Philips Res Rep Suppl 1973;5:1-80.

[11] Polder, D., Van Hove, M.. Theory of Radiative Heat Transfer between Closely Spaced Bodies. Phys Rev B 1971;4:3303-3314.

[12] Tsurimaki, Y., Chapuis, P.O., Okajima, J., Komiya, A., Maruyama, S., Vaillon, R.. Coherent regime and far-to-nearfield transition for radiative transfer. Journal of Quantitative Spectroscopy \& Radiative Transfer 2016 (submitted);. 
[13] Rytov, S.M.. Theory of Electric Fluctuations and Thermal Radiation. Bedford, MA: Air Force Cambridge Research Center; 1959.

[14] Rytov, S.M., Kravtsov, Y.A., Tatarski, V.I.. Principles of Statistical Radiophysics; vol. 3. Springer-Verlag; 1987.

[15] Narayanaswamy, A., Zheng, Y.. A Green's function formalism of energy and momentum transfer in fluctuational electrodynamics. Journal of Quantitative Spectroscopy and Radiative Transfer 2014;132:12-21.

[16] Biehs, S.A., Rousseau, E., Greffet, J.J.. Mesoscopic Description of Radiative Heat Transfer at the Nanoscale. Phys Rev Lett 2010;105(23):234301.

[17] Ordal, M.A., Bell, R.J., Alexander, R., Long, L., Querry, M.. Optical properties of fourteen metals in the infrared and far infrared: Al, co, cu, au, fe, pb, mo, ni, pd, pt, ag, ti, v, and w. Applied optics 1985;24(24):4493-4499.

[18] Ottens, R., Quetschke, V., Wise, S., Alemi, A., Lundock, R., Mueller, G., et al. Near-field radiative heat transfer between macroscopic planar surfaces. Phys Rev Lett 2011;107(1):14301.

[19] Kralik, T., Hanzelka, P., Zobac, M., Musilova, V., Fort, T., Horak, M.. Strong Near-Field Enhancement of Radiative Heat Transfer between Metallic Surfaces. Phys Rev Lett 2012;109(22):224302.

[20] Eyssa, Y., Okasha, O.. Thermodynamic optimization of thermal radiation shields for a cryogenic apparatus. Cryogenics 1978;18(5):305-307. 